\title{
An examination of 3D printing in dentistry - a study
}

\author{
Bekir Ciraka,* \\ aKaramanoglu Mehmetbey University, Department of Mechanical Engineering, TR-70001, Karaman/Turkey
}

\section{ART I C LE IN F O}

\section{Article history:}

Received 11 June 2018

Received in revised form 05 February 2019

Accepted 19 April 2019

Keywords:

3D Printing

Dentistry

Design

\begin{abstract}
A B S T RACT
This study examines the design and production processes of a small molar tooth produced with a 3D printer. When designing the exercises, polygons and parabolic forms were used as output points. These forms, which are rendered in three dimensions in the computer environment, are formed taking into consideration the structural characteristics of the production material. PLA (Polylactic Acid) material is used in this design which is produced in three dimensional printer by FDM (Fused Deposition Modeling) method. In the scope of the study, the conditions experienced in the design of the material used were mentioned and the material mistake was given during printing. The different effects of the commands used in the design process on the production surfaces, as well as voluntary or involuntary faults, are very important in the formation of some deformations and are caused by the plastic nature of the material. In addition, the effects of PLA on the surface of the faults are investigated. However, different methods applied in the production of sole parts and surfaces have been evaluated.
\end{abstract}

\section{Introduction}

Plastics are applied in the mold forming method especially in industrial production. Developments in three-dimensional printer technologies are used in areas such as design and crafts, cutting, joining, and so on. It is also used in traditional methods and techniques. In recent years, together with technologically advanced technologies, plastics can be produced using three-dimensional printers, and many workshops and artists are working on developing this technology and developing projects. These surveys, which are carried out at certain centers, enable significant quantities of production to be realized in the field. Some of these projects are managed academically in universities and some are under research. In this study, the dental production process which is produced with three-dimensional printer has been investigated [1].

\section{Design}

Rhinoceros 3D software was used in the design of bare teeth. The base portions of the vasors were solidified by the command "Extrcrv". $10 \mathrm{~cm}$ long, the working base is designed to be $20 \mathrm{~cm}$ in diameter. Lastly, the "Twist" command was applied and the teeth were finished with 45 degree angle. Completed work was recorded in the STL extension and the layer creation process was started. In this minor tooth design, solid model was created with Rhinoceros 3D and then processed on the surface using Meshmixer software. The The STL (Stereolithography) extension from Rhinoceros was opened in Meshmizer and the "Reduce Mesh" command was applied and the tooth surface (teberkül) was created with different percentages (Figure 1). 

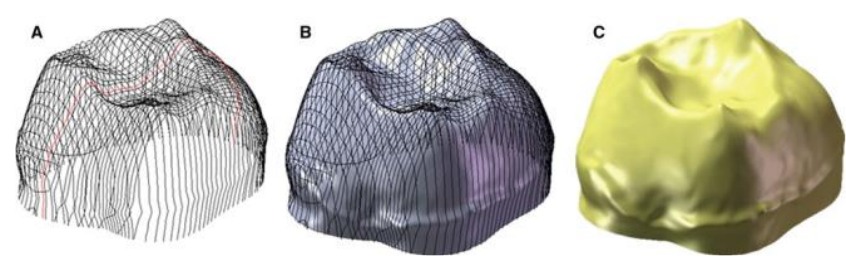

A - Mesh model B - Surface model C - Solid model

Figure1. CAD model levels

In order to be able to create different effects on the surface in the tooth design, some mistakes were intentionally applied during the design. Such mistakes allow the nozzle of the PLA material to be dispensed at the desired position. During prepress design phase, or during printing, such interventions have different effects on the surface. In some studies, the designer can intervene in the extruder or the table during the operation, revealing different designs and different workings made on the computer. In addition, rhythmic movements can be created by the sounds used during printing, and the printer can be intervened in this way. Other than this, errors that occur outside the control of the designer can also be encountered during printing. Such mistakes can lead to different occurrences [2].

\section{Creating Layers}

Cura software was used in the layer creation process. In the ceramic prints used, the distance between the layers is at most $1 \mathrm{~mm}$ and at least $0.5 \mathrm{~mm}$. The print speed was set at 40 $\mathrm{mm} / \mathrm{s}$ for all prints. The value is entered as 3 layers on the tooth base parts. In previous applications, it was observed that 2 or 1 layered bases were not suitable, cracks and spills were observed in the base parts after printing, after drying. In the base prints made as 4 and 5 layers, no deterioration was observed. However, it has been concluded that a 3-layered base is sufficient both to finish the printing in a shorter time and to prevent over thickness. No errors were observed in the prints made with a nozzle of $2 \mathrm{~mm}$ in diameter and 3 layers of the base due to the properties of the PLA material. (Figure 2)
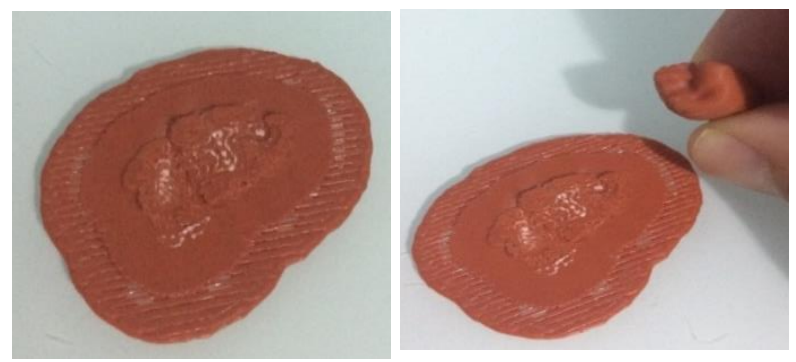

Figure 2. Creating layers of molar tooth in 3D printing

While the three-dimensional printing of the base parts is performed, some problems may occur during printing even if the number of layers is set correctly. This problem can be solved with a hard extruder that can repel the harder plastic consistency, or it can be solved with the adjustments made in the Cura software. When applied together with the nozzle thickness of $1 \mathrm{~mm}$ and Flow $100 \%$, which are standard values in the Cura's Basic section, the layer spacing is very close and the print is beginning to deteriorate when it is passed to a top layer. The thickness of the layer is $2 \mathrm{~mm}$ and the flow is $60 \%$ in the 3-dimensional prints made in the study and as mentioned above, the base is printed as at least 3 layers. No problems were encountered in the printing of the base parts in these values and successful results were obtained.

\section{3D Printing}

The prints were made with a three-dimensional delta printer. The period of printing for a tooth can be completed in $1.5-2$ hours depending on the size of the work. PLA feeds the extruder material of the printer with a pressure of usually 1.52 bar for the dental material. The video also shows how to print a 3D printer [3]. Here, due to the temperature difference that occurs during the printing process, the material freezes. To circumvent this situation, the periphery of the 3D printer shown in Fig. 3 is isolated.

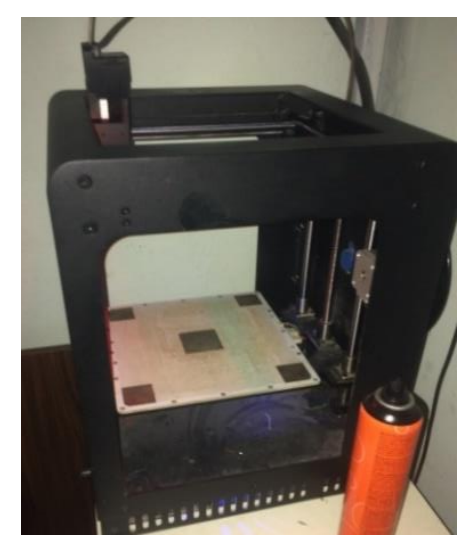

Figure 3. 3D printer

FDM technology has been used in 3D printer printing method. FDM is modeled by flowing the material to form layers of technology (Figure 4). The material used is PLA, a filament, rigid thermoplastic wrapped in rolls. The filament is thinned to a thickness of $2 \mathrm{~mm}$ through a hot nozzle tip and layers are formed with this thin plastic [4].

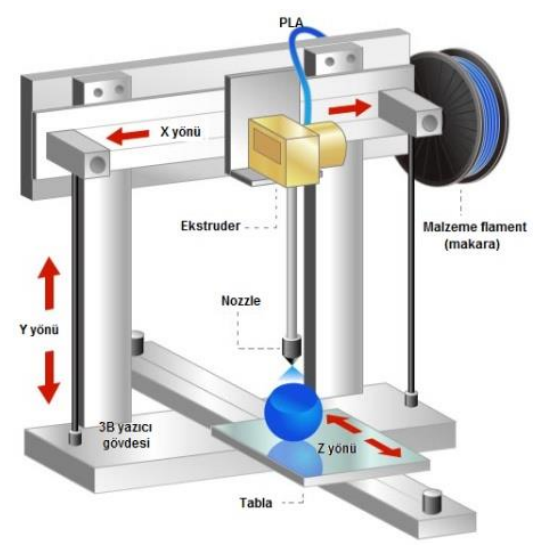

Figure 4. Part of 3D printer [6] 
Three dimensional printers continue to provide experimental results with increasingly reliable results. In these prints, the main problem is usually caused by the effects of gravity. But such problems also allow for experimental work when designed and implemented in a controlled manner. However, the fact that the material is not very soft and the setting of the ambient temperature also prevents such deformations [5].

After drying the $3 \mathrm{~B}$ prints, the base and mouth parts were retouched with a damp sponge to remove minor errors during printing. In three-dimensional prints, different color prints can be obtained by coloring the material. For this, it is firstly necessary to mix pigments withdifferent proportions at different rates. In Figure 5, the minor teeth finished with 3D printing are visible.
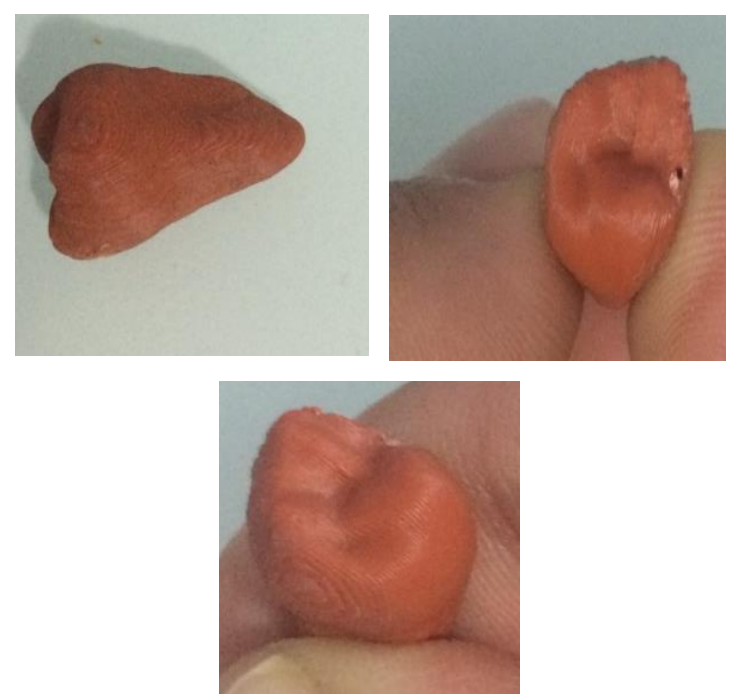

Figure. 5. Completed examines of molar tooth

\section{Conclusion}

In this study, it was concluded that a 3-layered floor was enough to finish both the printing in a shorter time and not to create excess thickness. No errors were observed due to the properties of the PLA material in the printing with a nozzle of $2 \mathrm{~mm}$ in diameter and a base of 3 layers.

\section{References}

[1].Bell, A., Ayoub, A.F., Siebert, P.: Assessment of the accuracy of a three-dimensional imaging system for archiving dental study models. J. Orthod. 30, 219-223 (2003)

[2].Davendorf, L. , Ryokai, K. "Beingthe Machine:
$\begin{aligned} & \text { ReconfiguringAgencyand } \\ & \text { Digital\&MaterialsFabrication, CHI 2015, Crossings, Seoul, Korea }\end{aligned}$

[3].Garino, F., Garino, G.B.: Comparison of dental archmeasurements between stone and digital dental casts. World J. Orthod. 3, 250- 254 (2002)

[4].Life of 3D Printer, Power point demo, Karabuk University,Department of Endustrial Design Engineering-2015.

[5].Quimby, M.L., Vig, K.W.L., Rashid, R.G., Firestone, A.R.: The accuracy and reliability of measurements madon computer-based digital models. Angle Orthod. 74, 298-303 (2004 Examines Videos of Molar Tooth in 3D Printer 